\title{
Effect of Saxagliptin, a Dipeptidyl Peptidase 4 Inhibitor, on Non-Alcoholic Fatty Liver Disease
}

This article was published in the following Dove Press journal:

Diabetes, Metabolic Syndrome and Obesity: Targets and Therapy

\author{
Lin Chen ${ }^{1, *}$ \\ Xiujuan Zhang $\mathbb{D}^{2, *}$ \\ Li Zhang ${ }^{3}$ \\ Dongmei Zheng ${ }^{2}$
}

'Department of Breast and Thyroid Surgery, Shandong Provincial Hospital, Cheeloo College of Medicine, Shandong University, Jinan, Shandong, People's Republic of China; ${ }^{2}$ Department of Endocrinology, Shandong Provincial Hospital Affiliated to Shandong University, Shandong Provincial Hospital Affiliated to Shandong First Medical University, Jinan, People's Republic of China; ${ }^{3}$ Department of Vascular Surgery, Shandong Provincial Hospital Affiliated to Shandong University, Shandong First Medical University \& Shandong Academy of Medical Sciences, Jinan, People's Republic of China

*These authors contributed equally to this work
Correspondence: Dongmei Zheng Department of Endocrinology, Shandong Provincial Hospital Affiliated to Shandong University, Shandong Provincial Hospital Affiliated to Shandong First Medical University, Jinan, Shandong Province 25002I, People's Republic of China Tel + 8653168776375

Email zhengdongmeil97I@I63.com
Background and Aim: Non-alcoholic fatty liver disease (NAFLD) represents a broad spectrum of chronic liver disease characterized by aberrant accumulation of triglycerides (TG) in hepatocytes without excessive alcohol consumption. Hepatic lipotoxicity derived from overaccumulation of free fatty acids is considered as one of the typical hallmarks of NAFLD. Insulin resistance (IR) and chronic inflammation are widely recognized as the key etiological factors associated with NAFLD. Dipeptidyl peptidase 4 inhibitor (DPP4i) is a novel pharmacological agent extensively applied in the treatment of Type 2 Diabetes Mellitus (T2DM) for decades which also have a liver protective effect.

Methods: In order to invest the therapeutic efficiency and underlying mechanism of DPP4i saxagliptin, we used high-fat diet (HFD) and streptozotocin-induced NAFLD treated with saxagliptin. Biochemical, histomorphological, genetic and protein expression of related pathways were investigated.

Results: Fasting blood glucose (FBG), TG, total cholesterol (TC), and low-density lipoprotein cholesterin significantly increased in NAFLD group, which also exhibited severe steatosis. Other remarkable findings were hyperinsulinemia, increased DPP4, PTP-1B and $T N F-\alpha$ level and decreased GLP-1, $A C O X-1, C P T-1 A$ expression, concomitant with liver DPP4 expression enhancement and serum DPP4 elevation. These undesirable consequences were alleviated by saxagliptin to a certain degree.

Conclusion: DPP4i saxagliptin improves NAFLD by ameliorating IR, inflammation, downregulation of hepatic DPP4 and sDPP4, as well as subsequent steatosis. The elevation of hepatic DPP4 and sDPP4 and succedent post-treatment decrease suggested that DPP4 may involve in the development of NAFLD. The anti-lipotoxic effect of DPP4i may involve the activation of $C P T 1 A$ and $A C O X 1$ related $\beta$-oxidation signaling pathway suppression of $T N F-\alpha$ mediated inflammatory and $P T P-1 B$. The results covered in this article showed that saxagliptin affects many aspects of the pathological characteristics of NAFLD, suggesting that DPP4i saxagliptin may offer a novel therapeutic option for NAFLD.

Keywords: dipeptidyl peptidase 4 inhibitor DPP4i, non-alcoholic fatty liver disease NAFLD, inflammation, insulin resistance, IR

\section{Introduction}

Non-alcoholic fatty liver disease (NAFLD) represents a broad spectrum of liver disease encompassing non-alcoholic fatty liver, non-alcoholic steatohepatitis, irreversible fibrosis, cirrhosis and hepatocellular carcinoma. ${ }^{1}$ Due to rapid lifestyle changes, the prevalence of NAFLD in China has increased from $18 \%$ to $29 \%$, in parallel with soaring diagnoses of diabetes. ${ }^{2,3}$ It is conjectured that China will have the world's highest prevalence of NAFLD, with 314.58 million cases by $2030 .^{4}$ The pooled 
prevalence of NAFLD in Type 2 Diabetes Mellitus (T2DM) patients is even higher, estimated to be $59.67 \%{ }^{5}$

It has been generally acknowledged that NAFLD start with the aberrant accumulation of triglycerides (TG) in hepatocytes in the absence of excessive alcohol consumption. Steatosis may occur along with portal and lobular inflammation as well as hepatocyte injury, which are highly likely progress to hepatocellular carcinoma. ${ }^{6}$

Although the precise mechanisms involved in the pathogenesis of NAFLD has not been fully elucidated, insulin resistance (IR), chronic inflammation, and T2DM are widely recognized as the key etiological factors. ${ }^{7}$ Domestic and overseas researchhave revealed that ERS is also closely related to NAFLD. ${ }^{8}$

IR is defined as the underreaction of cells to physiological levels of insulin, which plays a critical role in the development of NAFLD. Plenty of epidemiological and therapeutic evidence indicate that IR is the primary mechanism of hepatic steatosis. Dipeptidyl peptidase 4 (DPP4) is a multifunctional serine protease which exerts pleiotropic effects on lipid metabolism by direct action and inactivation of incretin such as glucagon-like protein 1 (GLP-1). ${ }^{9}$ Simultaneously, elevated DPP4 expression and activity have been proved to play an auxo-action for the occurrence and severity of NAFLD. ${ }^{10}$

NAFLD has become a very real problem which has a crucial impact on public health and the economy. Despite the continuous progress and evolution made in the treatment of NAFLD, there still lacks an effective means of controlling NAFLD in advance.

Dipeptidyl peptidase 4 inhibitor (DPP4i) is a novel pharmacological agent extensively adhibited in the treatment of T2DM for decades, as it lower blood glucose level through inactivation of incretin peptides glucose-dependent insulinotropic polypeptide (GIP) and glucagon-like peptide-1 (GLP-1). Multiple clinical trials have reported that it could improve hepatic insulin sensitivity, downregulate fibroblast growth factor, ${ }^{11}$ suppress hepatic inflammation, and ameliorate fatty acid oxidation. ${ }^{12,13}$ These peculiarities indicate that DPP4i may be applicable for NAFLD.

DPP4i saxagliptin has been reported to improve hepatocyte steatosis among T2DM patients who were newly diagnosed with NAFLD. ${ }^{14}$ Therefore, we assessed the therapeutic effect and related mechanism of saxagliptin using a high-fat diet (HFD) combined with STZ injection induced NAFLD model.

\section{Methods}

\section{Animals}

Thirty male Sprague-Dawley rats weighing 130-150g (SLAC Laboratory Animal Co., Ltd) were housed in a constant temperature-controlled room, with a $12 \mathrm{~h}$ light/ dark cycle and free access to food and water. During the process of the experiment, the rats' general condition and body weight were monitored weekly. The experiments were performed in strict accordance with the Shandong Provincial Hospital Animal Care and Use Committee to minimize the number of animals used and their suffering. All animals used in this study were cared for in accordance with the recommendations in the Guide for Laboratory Animal - guideline for ethical review of animal welfare (GB/T 35892-2018). All experimental protocols were approved by the Animal Ethics Committee of Shandong Provincial Hospital.

\section{Experimental Design}

After 1 week of adaptive feeding, the rats were randomly divided into a normal control group (NC group, $\mathrm{n}=10$ ) and a NAFLD group (NAFLD group, $n=20$ ), each group was assigned to one of the following diets respectively: 1) NC diet: $100 \%$ standard rodent chow; 2) HFD: $82.3 \%$ standard rodent chow supplemented with 5\% sucrose, $10 \%$ lard, $2 \%$ cholesterol, $0.5 \%$ sodium cholate and $0.2 \%$ propylthiouracil. ${ }^{15}$ Joint combination of an HFD and low-dose injection of streptozotocin were used to induce diabetic rats with NAFLD in this research, since it better resembles the human pathology of T2DM and NAFLD. ${ }^{16}$ After 4 weeks, NAFLD rats were administered with 30 $\mathrm{mg} / \mathrm{kg}$ of streptozotocin with sodium citrate buffer $(\mathrm{pH}=4.5)$ through a single peritoneal cavity injection, whereas $\mathrm{NC}$ group rats were injected with same volume of buffer. At the end of 8 weeks, two rats were randomly decapitated from experimental group to verify the morphological changes of liver. Meanwhile, fasting blood glucose (FBG) was measured from tail vein, rats showing FBG values of $\geq 11.1 \mathrm{mmol} / \mathrm{L}$ were considered as a successfully established T2DM associated NAFLD model. NAFLD group was then randomly divided into control group (NAFLD-C, $\mathrm{n}=9$ ) and treatment group (NAFLD-T, $\mathrm{n}=8$ ), simultaneously, equal volume of saxagliptin (30mg/ $\mathrm{kg}$ per day) and normal saline were dosed to the treatment group and control group respectively by means of intragastric administration. 
Table I Primers of qRT-PCR

\begin{tabular}{|l|l|l|l|}
\hline Gene & Genbank ID & Primer Sequence & Product Size(bp) \\
\hline TNF $\alpha$ & 24,835 & $\begin{array}{l}\text { F:5' CACCACGCTCTTCTGTCTACTG 3' } \\
\text { R:5' GGCTACGGGCTTGTCACTC 3' }\end{array}$ & 148 \\
\hline PTPIB & 24,697 & $\begin{array}{l}\text { F:5' TCAGCCCTTTTGACCACAGT 3' } \\
\text { R:5' CCACGCCCCTGCTCTTC 3' }\end{array}$ & 177 \\
\hline CPTI & 25,757 & $\begin{array}{l}\text { F:5'GAGCCACGAAGCCCTCAAAC 3' } \\
\text { R:5'CACACCCACCACCACGATAAG 3' }\end{array}$ & 139 \\
\hline AcoxI & $50,68 I$ & $\begin{array}{l}\text { F:5' AATCAAGCAAAGCGAACCAG 3' } \\
\text { R:5' GCCTTCAGCCCAGCAGTG 3' }\end{array}$ & 204 \\
\hline
\end{tabular}

\section{Specimen Collection and Index Detection}

Fasting tail vein blood sampling were collected at the end of week 4, 8 and 12, for the measurement of FBG, TG, total cholesterol (TC) and low-density lipoprotein cholesterin (LDL-C). At the end of the experiment, all mice were decapitated after overnight fasting. Subsequently, the liver were immediately dissected and weighed. The liver index was calculated according to the following formula: Liver index $=($ liver wet weight/body weight $\times 100 \%)$. A piece of liver tissue from the middle part of right lobe was fixed in paraformaldehyde solution, which was later used for morphological examination. The residual liver tissue was frozen immediately at $-80^{\circ} \mathrm{C}$ for the qRT-PCR.

\section{ELISA Analysis}

The concentrations of GLP-1, insulin and DPP4 in plasma were determined by ELISA method with commercially available assay kits (GLP-1:CEA804Ra; DPP4: SEA884Ra; Insulin:GR150359-1).

\section{Immunohistochemistry}

Excised liver tissues were sectioned and deparaffinized, then endogenous peroxidase was blocked and inactivated. After microwave antigen retrieval with citric acid buffer (PH6.0), slides were blocked with premium quality normal goat serum. Whereafter, the primary antibody: DPP4 antibody (ab28340, 1:200) was added and incubated overnight, the secondary goat anti-rabbit IgG antibody was applied followed by the instillment of streptavidin-peroxidase. Negative control staining was performed by the substitution of the primary DPP4 antibody with a PBS buffer solution. At length, the cells exhibited brown by means of the 3,3'- Diaminobenzidine (DAB) staining techniques combined with counterstaining using hematoxylin. Staining intensity was classified in scores on a scale of
0-3: 0: no obvious coloration; 1: slightly yellow; 2 : tan or deep yellow; 3: sepia or black brown. The percentage of the positive cells was classified in scores on a scale of $0-4$ : 0: $0-5 \%$; 1: 6 25\%; 2: 26 50\%; 3: 51 75\%; 4: >75\%.

\section{Western Blot Analysis}

Liver tissue samples were homogenized and centrifuged, then the supernate were collected. The protein concentrations were determined using the bicinchoninic acid kit. After supplemented with the loading buffer and boiled, total proteins were separated by SDS-PAGE and transferred to PVDF membrane. The membrane was blocked subsequently incubated with primary antibodies against $A C O X 1$ (sc-107375, Santa Cruz Biotechnology, Inc.), PTP1B, TNF$\alpha$ and CPT1A (ab52650; ab66579; ab128568). The membrane was washed and incubated with secondary antibody and electrogenerated chemiluminescence. Band densities were quantified using Image $\mathrm{J}$ program.

\section{Biochemical Analysis}

Serum TC, TG and LDL-C were assessed by commercial kits following the standard procedure, including TC Assay Kit, TG Kit, LDL-C Kit (006301, 006304, 006340; Beijing BHKT Clinical Reagent Co. Ltd., China).

Table 2 Effect of Treatment and Diets on Nutrition Status

\begin{tabular}{|l|l|l|l|}
\hline & NC & NAFLD-C & NAFLD-T \\
\hline $\begin{array}{l}\text { Body weight (g) } \\
8 \text { weeks }\end{array}$ & & & \\
10 weeks & $494.88 \pm 37.36$ & $400.40 \pm 39.08$ & $402.50 \pm 25.41$ \\
12 weeks & $428.67 \pm 39.93$ & $479.00 \pm 33.35^{*}$ & $442.82 \pm 32.53$ \\
\hline Liver weight (g) & $11.44 \pm 1.25$ & $16.65 \pm 1.98^{* *}$ & $16.78 \pm 0.69$ \\
Liver index (\%) & $2.69 \pm 0.43$ & $3.47 \pm 0.23^{* *}$ & $3.80 \pm 2.59$ \\
\hline
\end{tabular}

Notes: $* \mathrm{P}<0.05, * * \mathrm{P}<0.01$. 
Quantitative Real-Time Polymerase Chain Reaction

Total RNA was extracted from liver by Trizol method using the RNApure total RNA rapid isolation kit (GK3016; Shanghai Generay Biotech Co., Ltd.). The purity and concentration of the RNA was measured. cDNA was obtained using the RevertAid First Strand cDNA synthesis Kit (K1622; Thermo Fisher Scientific, UK). Primer sequences are presented in Table 1. Results were expressed as the relative values after normalization to GAPDH.

\section{Hematoxylin and Eosin (H\&E) Staining}

Liver tissues were fixed, embedded and then sliced into 4 $\mu \mathrm{m}$ thick sections. The sections were stained with HE for histopathologic analysis. Each section was randomly selected for 3 fields, which were used to gauge hepatic steatosis (steatotic cell number/total cell number) on a
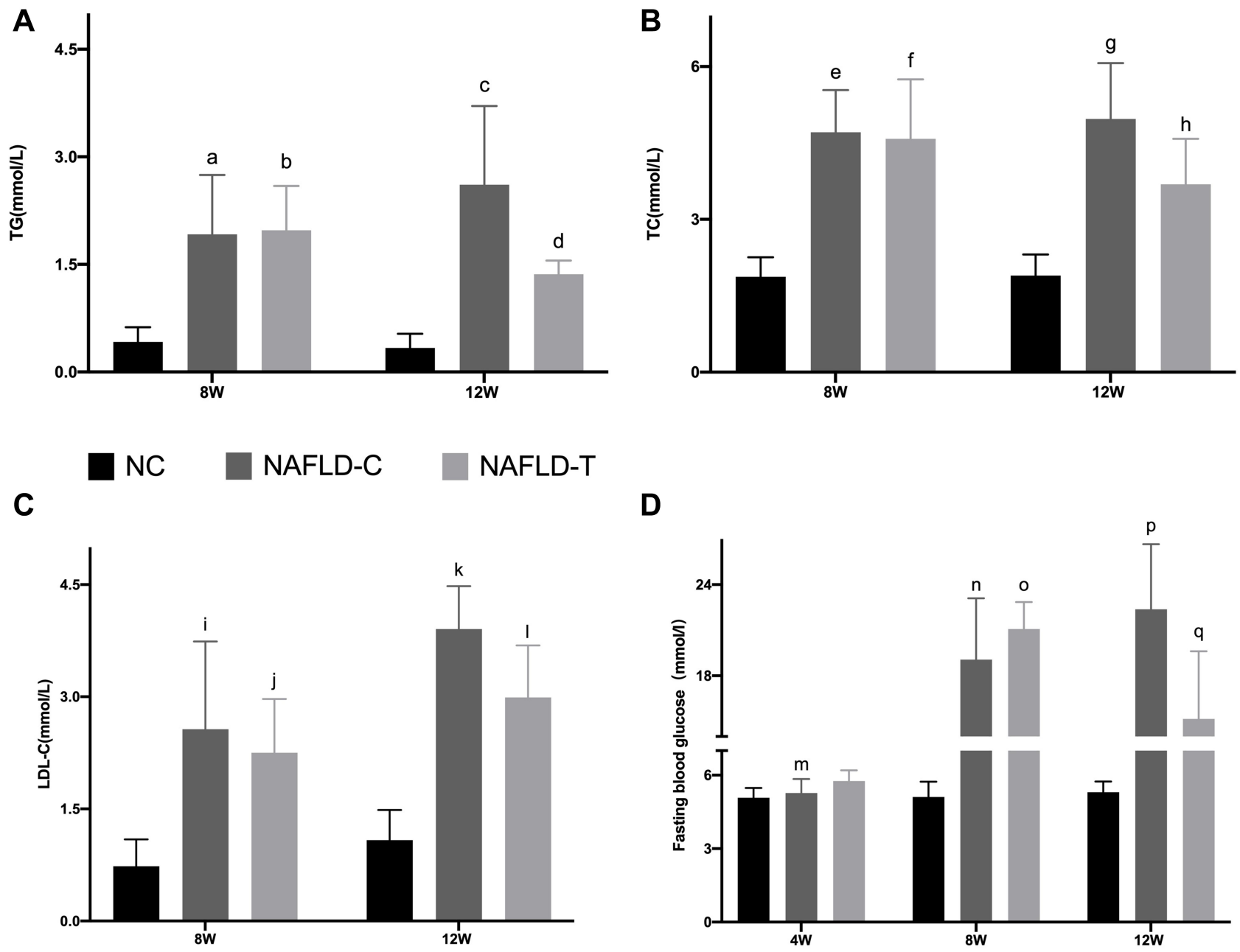

Figure I Effect of DPP4i on serum (A) TG, (B) TC, (C) LDL-C, (D) FBG level in NAFLD mice. a: no significant difference between NAFLD-C (8w) and NAFLD-T group $(8 w)$; no significant difference between NAFLD-C group ( $8 w)$ and NAFLD-C group (I2w); NAFLD-C group (8w) vs. NC group (8w) P < 0.01 . b: NAFLD-T group (8w) vs. NC group (8w) P < 0.0I; NAFLD-T group (8w) vs. NAFLD-T group (I2w) P < 0.05. c: NAFLD-C group (I2w) vs. NC group (I2w) P < 0.01 ; NAFLD-C group (I $2 w)$ vs. NAFLD-T (12w) P < 0.05. d: NAFLD-T group (12w) vs. NC group $(12 w) P<0.05$. e: no significant difference between NAFLD-C (8w) and NAFLD-T group (8w); no significant difference between NAFLD-C group ( $8 w$ ) and NAFLD-C group ( $12 w)$; NAFLD-C group ( $8 w)$ vs. NC group ( $8 w$ ) P < 0.01 . f: NAFLD-T group ( $8 w$ ) vs. NC group $(8 w)$ P < 0.0I; no significant difference between NAFLD-T group ( $8 w$ ) and NAFLD-T group (I2w). g: NAFLD-C group (I2w) vs. NC group (I2w) P < 0.00I; NAFLD-C group $(12 w)$ vs. NAFLD-T $(12 w) P<0.05$. h: NAFLD-T group $(12 w)$ vs. NC group $(12 w) P<0.05$. i: no significant difference between NAFLD-C (8w) and NAFLD-T group (8w); NAFLD-C group ( $8 w$ ) vs. NAFLD-C group ( $12 w) P<0.05$; NAFLD-C group ( $8 w)$ vs. NC group (8w) P $<0.00 I$. j: NAFLD-T group $(8 w)$ vs. NC group $(8 w) P<0.05$; no significant difference between NAFLD-T group ( $8 w)$ and NAFLD-T group $(12 w)$. k: NAFLD-C group $(12 w)$ vs. NC group (I2w) P < $0.00 \mid$; NAFLD-C group (I $2 w)$ vs. NAFLD-T (I $2 w)$ P < 0.05. I: NAFLD-T group $(12 w)$ vs. NC group $(12 w) P<0.00 I$. m: no significant difference between NAFLD-C (4w) and NAFLD-T group (4w) and NC group (4w). n: NAFLD-C group ( $8 w)$ vs. NC group ( $8 w) P<0.00$ I; NAFLD-C group (4w) vs. NAFLD-C group ( $8 w) P<0.05$; no significant difference between NAFLD-C $(8 w)$ and NAFLD-T group (8w). o: NAFLD-T group (4w) vs. NAFLD-T group (8w) P < 0.05; NAFLD-T group (8w) vs. NC group (8w) P < $0.00 \mathrm{I}$. p: NAFLD-C group (I2w) vs. NC group (I2w) P < 0.00I; NAFLD-C group (I2w) vs. NAFLD-T group (I2w) P<0.0I; no significant difference between NAFLD-C group (I2w) and NAFLD-C group (8w). q: NAFLD-T group (8w) vs. NAFLD-T group (I2w) P < 0.05; NAFLD-T group (I2w) vs. NAFLD-C group (I2w) P $<0.01$; NAFLD-T group (I2w) vs. NC group (I2w) $P<0.001$. 
scale of 0 to 4: 0 : no obvious degeneration; $1: \leq 25 \% ; 2$ : 25 50\%; 3: 50 75\%; 4: $>75 \%$.

\section{Statistical Analysis}

All data were expressed as Mean \pm SD. $\mathrm{P}<0.05$ were considered statistically significant. Statistical analyses were conducted using SPSS statistical software version 25. Statistical significance was determined by one-way ANOVA for multiple comparisons with Dunnett's post hoc test.

\section{Results}

\section{Effect of DPP4i on Nutrition Status, Serum Lipid and Glucose Levels}

During the 12 week feeding period, body weight, liver weight and liver index (Table 2) in NAFLD-C group were significantly higher than NC group, meanwhile, body weight and liver weight showed a certain degree of decrease in posttreatment group. As the duration of the feeding time was prolonged, serum TG ( $p<0.01)$, LDL-C, TC ( $p<0.01)$ and FBG levels showed a statistically significant increasing trend in HFD rats. After being administrated with DPP-4i, serum TG $(p<0.05)$ levels dramatically reduced in the NAFLD-T group compared with NAFLD-C group (Figure 1A). Although the result did not reach statistical significance, TC showed a downward trend after treatment (Figure 1B). Compared to 4 weeks previously, LDL-C level $(\mathrm{p}<0.05)$ remarkably increased in the NAFLD-C group at 12 weeks. On the other hand, DPP-4i significantly reduced LDL-C level (Figure $1 \mathrm{C}, \mathrm{p}<0.05$ ). Elevation in FBG values averaged $15 \mathrm{mmol} / \mathrm{l}$ above normal (Figure 1D). In comparison with the gradual deterioration of FBG in the untreated NAFLD-C group $(\mathrm{p}<0.01)$ in the previous 4 weeks, this sharp increase was prominently attenuated by DPP-4i in the treatment group $(\mathrm{p}<0.05)$ plateauing $7 \mathrm{mmol} / \mathrm{l}$ below the untreated controls.

\section{Effects of DPP4i on Plasma GLP-I, DPP4 and Insulin Level}

Serum GLP-1 levels in NAFLD rats were lower than normal conditions at 8 weeks (Figure 2A, p <0.001). GLP-1 level was statistically improved after 4 weeks of DPP4i treatment $(\mathrm{p}<0.01)$. Serum insulin (Figure $2 B$ ) and DPP4 (Figure 2C) levels in the model group were higher than NC group at 8 weeks $(p<0.001)$. Saxagliptin significantly reduced serum DPP4 levels $(\mathrm{p}<0.05)$, alleviated IR. However, serum insulin level slightly decreased which did not reach statistical significance.

\section{Effects of DPP4i on Liver DPP-IV Expression}

A semi-quantitative score system was used to classify DPP4 expression in the liver based on the staining
A

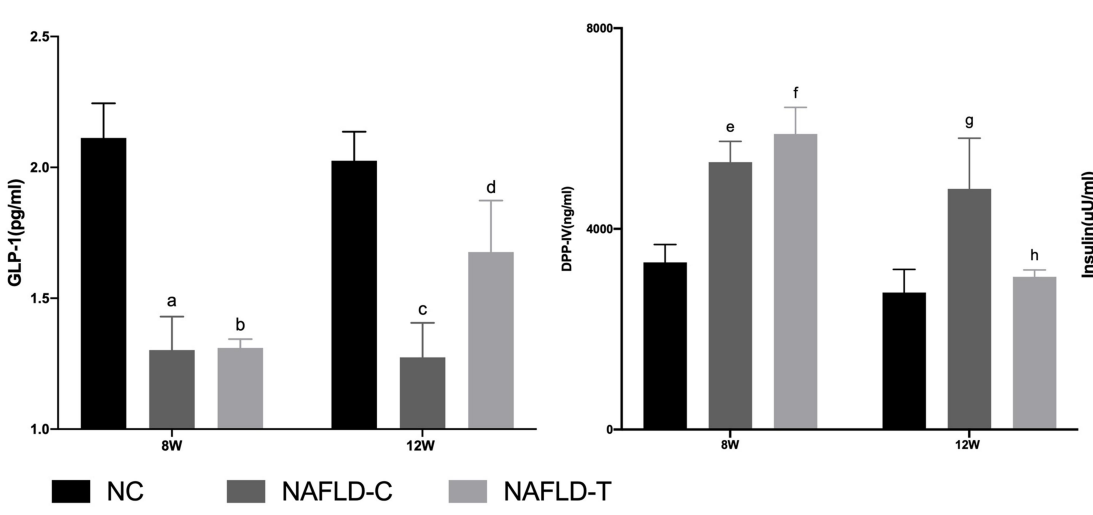

C

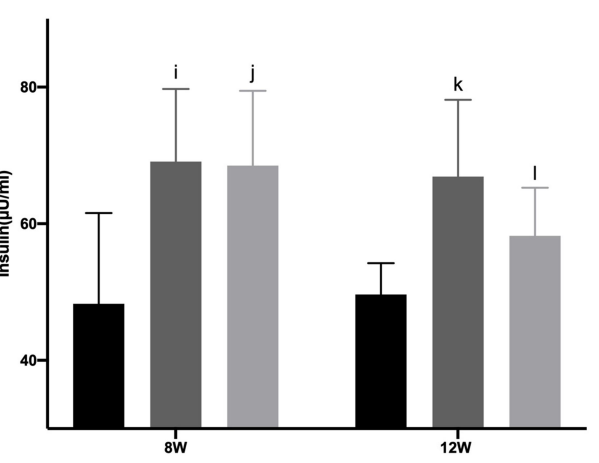

Figure 2 (A) Effect of DPP4i on serum GLP-I level in NAFLD mice. (B) Effect of DPP4i on serum DPP4 level in NAFLD mice. (C) Effect of DPP4i on serum insulin level in NAFLD mice. a: no significant difference between NAFLD-C (8w) and NAFLD-T group (8w); no significant difference between NAFLD-C group (8w) and NAFLD-C group (12w); NAFLD-C group (8w) vs. NC group (8w) P < 0.00I. b: NAFLD-T group (8w) vs. NC group (8w) P<0.00I; NAFLD-T group (8w) vs. NAFLD-T group (I2w) P< 0.01 .

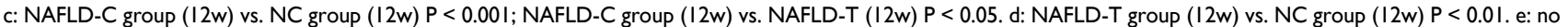
significant difference between NAFLD-C $(8 w)$ and NAFLD-T group ( $8 w)$; no significant difference between NAFLD-C group (8w) and NAFLD-C group (I $2 w)$; NAFLD-C group (8w) vs. NC group (8w) P < 0.00I. f: NAFLD-T group (8w) vs. NC group (8w) P < 0.00I; NAFLD-T group (8w) vs. NAFLD-T group (I2w) P < 0.05. g: NAFLD-C

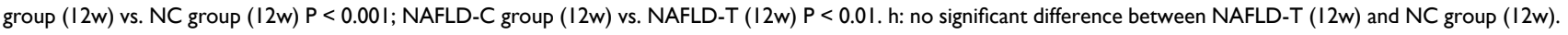
i: no significant difference between NAFLD-C (8w) and NAFLD-T group (8w); no significant difference between NAFLD-C group (8w) and NAFLD-C group (I $2 w)$; NAFLD$C$ group ( $8 w)$ vs. NC group (8w) P < 0.05. j: NAFLD-T group (8w) vs. NC group ( $8 w$ ) P < 0.05; no significant difference between NAFLD-T group (8w) and NAFLD-T group $(12 w)$. k: NAFLD-C group (I $2 w)$ vs. NC group (I2w) P < 0.01 ; no significant difference between NAFLD-C group (I2w) and NAFLD-T group (I2w). I: no significant difference between NAFLD-T (I2w) and NC group (I2w). 


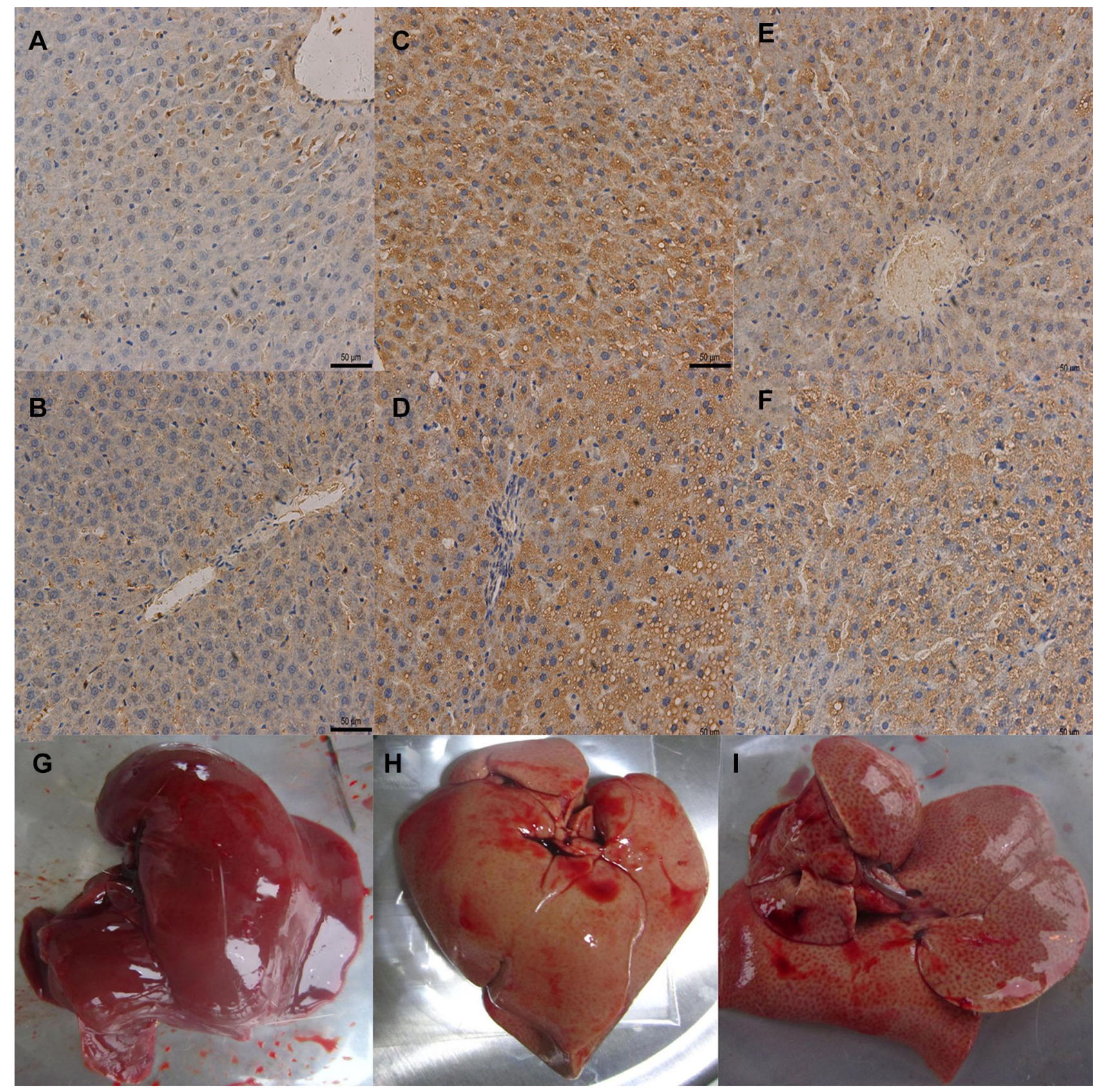

Figure 3 (A-B) Effect of DPP4i treatment on liver DPP4 expression in NC group (×200); (C-D) Effect of DPP4i treatment on liver DPP4 expression in NAFLD-C group ( $\times 200)$; (E-F) Effect of DPP4i treatment on liver DPP4 expression in NAFLD-T group ( $\times 200)$; (G) Gross specimens of NC group, (H) Gross specimens of NAFLD-C group, (I) Gross specimens of NAFLD-T group.

intensity and percentage of positive cells: 0 score: negative; 1-6 scores: weakly positive; 7-12 scores: strongly positive. The expression levels of DPP-IV between NC group and NAFLD-C group, between NAFLD-C group and NAFLD-T group showed statistically significant differences (Figure 3A-F, Figure 4A, Table 3). Furthermore, DPP-IV strongly positive expression rate of NAFLD-C group were $93.3 \%$, average scoring value was 10.60 \pm 2.13 , after treatment, DPP-IV strongly positive expression rate were significantly reduced to $46.7 \%$, average scoring value is $6.87 \pm 2.33$. Together, DPP4 was overexpressed in NAFLD, and DPP4 was significantly diminished after saxagliptin treatment. Comparatively speaking, DPP-4i resulted in approximately 2 -fold diminution of DPP4 strongly positive expression rate in treatment group. 

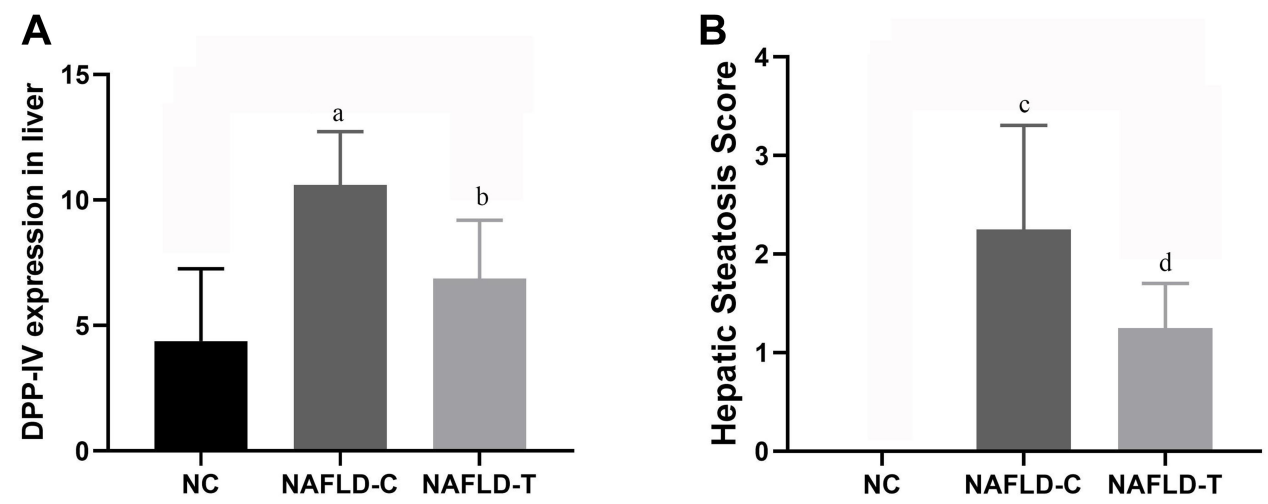

Figure 4 (A) Effect of DPP4i treatments on liver DPP4 expression, our results showed that DPP4 was overexpressed in non-alcoholic fatty liver tissue and DPP4 was significantly diminished after saxagliptin treatment. (B) Effect of DPP4i treatments on hepatic steatosis score. a: NAFLD-C group vs. NC group $P<0.0$ I; b: NAFLD-C group vs. NAFLD-T group $P<0.05$; c: NAFLD-C group vs. NC group $P<0.0$; d: NAFLD-C group vs. NAFLD-T group $P<0.05$.

\section{Effects of DPP4i on Liver Morphology and Histological}

The liver specimens of NC group (Figure 3G) were moist, glossy and resilient. By contrast, the livers of the NAFLD group (Figure 3H-I) were enlarged, lacklustre and had pale discoloration on the surface. For healthy rats (Figure 5A and B), hepatic cells radially encircled the central vein, and there was no obvious degeneration or necrosis of hepatocytes. In Figure 5C-F, the sections from the model group exhibited moderate to severe injury characterized by varying degrees of fatty denaturation and inflammatory cell infiltration, which were in accordance with the biochemical index differences.

The NAFLD-C group displayed widespread microvesicular fat deposition as well as minor punctate inflammation, the average scoring value was $2.25 \pm 1.06$. In comparison, liver injury in the DPP-4 inhibitor treated NAFLD-T rats was mitigated dramatically, the average scoring value was $1.25 \pm 0.45$ (Figure 4B, Table 3).

\section{Effects of DPP4i on Inflammatory Hepatic Cytokines and Hepatic Metabolic Genes}

We measured the expressions of $A C O X 1, C P T 1 A, T N F-\alpha$ and $P T P 1 B$ in the liver. As shown in Figure 6, significant

Table 3 Histopathological Features

\begin{tabular}{|l|l|l|l|}
\hline & NC & DM-T & DM-C \\
\hline $\begin{array}{l}\text { DPP-IV expression score } \\
\text { (M士SD) }\end{array}$ & $4.38 \pm 2.89$ & $6.87 \pm 2.33$ & $10.60 \pm 2.13$ \\
\hline $\begin{array}{l}\text { DPP-IV Positive rate } \\
\text { Strongly positive rate } \\
\text { Weakly positive rate }\end{array}$ & $20.8 \%$ & $46.7 \%$ & $93.3 \%$ \\
\hline Hepatic steatosis score & $0.00 \pm 0.00$ & $2.25 \pm 1.06$ & $1.25 \pm 0.45$ \\
\hline
\end{tabular}

increase in the mRNA expression of $T N F-\alpha$ was shown in the livers of NAFLD-C mice compared with NC mice. Saxagliptin significantly reduced the mRNA and protein expression of $T N F-\alpha$. In addition, significant reduction of $\beta$-oxidation related genes $A C O X-1$ and $C P T-1 A$ were observed in the NAFLD-C group. The alternation in CPT-1A mRNA and protein levels were significantly prevented by DPP4i administration. DPP4i could partially improve $A C O X-1$ mRNA reduction by $37 \%$, which did not reach statistical levels. In the current study, enhanced mRNA expression of PTP $1 B$ was observed in NAFLD-C group, its expression was 2.77 fold higher than $\mathrm{NC}$ group. Saxagliptin significantly downregulated $P T P 1 B$ expression by $13.1 \%$ (2.41-fold), which was also observed in protein expression.

\section{Discussion}

NAFLD refers to the abnormal fatty acid infiltration in liver without excessive alcohol consumption. Importantly, NAFLD is an escalating health issue which afflicts around $25.24 \%$ of the general population in the worldwide context. ${ }^{1}$ Moreover, NAFLD patients usually exhibit nonspecific symptoms, such as fatigue and hepatic region discomfort, which makes early detection difficult. Therefore, NAFLD has attracted more and more attention both at home and abroad due to its high incidence, obscure symptoms and liver-related detrimental outcomes. The exact mechanism underlying its onset and progression is intricate. Previous researchhas emphasized the multifactorial interaction between metabolic syndrome, T2DM and NAFLD. Notably, the association between NAFLD and T2DM has been well established, ${ }^{17}$ which could synergistically generate adverse outcomes including higher stage steatosis and more serious complications. 


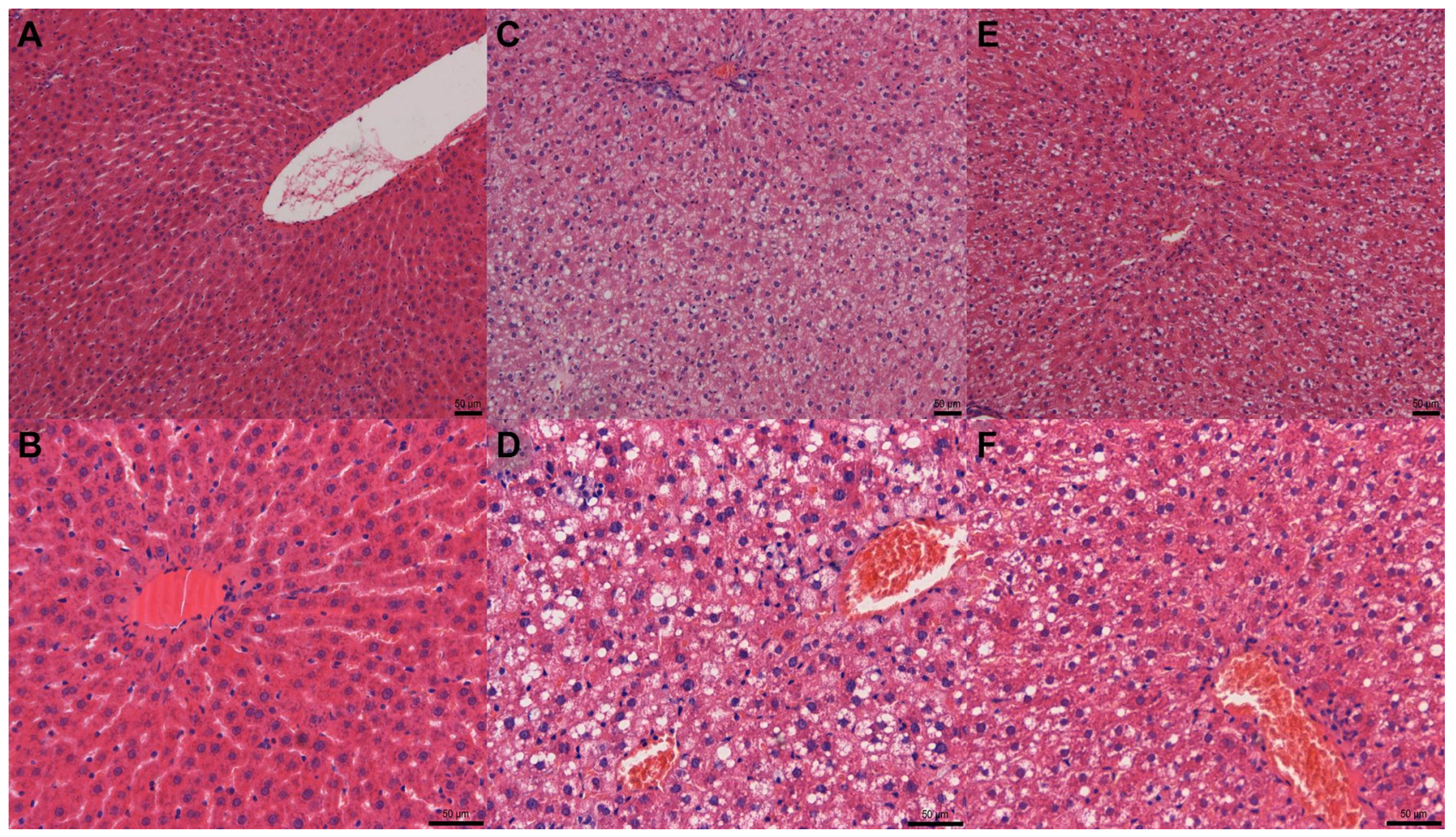

Figure 5 Effect of DPP4i treatment on liver steatosis. Sections were stained with hematoxylin \& eosin. (A-B) histologic pattern of NC group (original magnification $\times 100$, $\times 200$ respectively); (C-D) histologic pattern of NAFLD-C group (original magnification $\times 100, \times 200$ respectively); (E-F) histologic pattern of NAFLD-T group (original magnification $\times 100, \times 200$ respectively).

In this study, the HFD yielded overweight IR mice with hypertrophied livers, together with liver steatosis. Other remarkable findings were hyperglycemia, hyperlipidemia, hyperinsulinemia, increased DPP4, PTP- $1 B$ and $T N F-\alpha$ level and decreased GLP-1, ACOX-1, CPT-1A expression, concomitant with liver DPP4 expression enhancement and serum DPP4 elevation. These undesirable consequences were alleviated by saxagliptin.

There are considerable evidence implicating that oxidative stress and inflammatory response are the two broad categories participating in the development of NAFLD except for IR. ${ }^{18}$ IR is defined as the underreaction of cells to physiological levels of insulin. ${ }^{19}$ Plenty of epidemiological and therapeutic evidence indicates that IR is the shared origin of both NAFLD and T2DM, leading to disease aggravation in a vicious cycle. ${ }^{20}$ IR could upregulate hepatic de novo lipogenesis (DNL) and lipolysis, reduce $\beta$ oxidation and saturated fatty acid consumption, which eventually accelerate the release and influx of free fatty acid. Acyl-CoA oxidase 1 (ACOXI) is the rate limiting enzyme, participating in the first step of $\beta$-oxidation, which catalyzes the desaturation of acyl-CoAs. ${ }^{21}$ Carnitine palmitoyl transferase-IA (CPT-1A) involves in the translocation across inner mitochondrial membrane. ${ }^{22} A C O X 1$ and $C P T-1 A$ are crucial transcriptional regulators of the FA $\beta$-oxidation pathway. ${ }^{23}$ In the current study, NAFLD rats showed significantly reduced $C P T-1 A$ expression in association with increased serum TG level, which were significantly alleviated by saxagliptin. However, there was no obvious effect of saxagliptin on the diminution of $A C O X 1$ mRNA. On the one hand, improved expression of CPT-1A not only increases $C P T-1 A$ activity but also decreases hepatic TG content, which thereby increases fatty acid oxidation capacity. ${ }^{24}$ On the other hand, saxagliptin could relieve hepatic steatosis directly caused by $C P T-1 A$ dysfunction. ${ }^{25}$ Therefore, it can be conjectured that saxagliptin ameliorates hepatic steatosis by hepatic $C P T-1 A$ activation.

Working as an inflammatory cytokine, Tumor necrosis factor- $\alpha(T N F-\alpha)$ has been postulated as an essential mediator of IR in the pathogenesis of NAFLD. Notably, $T N F-\alpha$ level is also in accordance with the histological severity of NASH. ${ }^{26}$ In our study, NAFLD rats exhibited remarkable elevation of $T N F-\alpha$ mRNA and protein expression as well as hepatic steatosis, which were significantly relieved by saxagliptin. Exceptional lipid accumulation, caused by upregulation of serum TG level and hepatic DNL, ${ }^{27}$ sensitize both steatotic and normal hepatocytes, triggering cytotoxic and apoptotic mechanism. ${ }^{28}$ Under this circumstance, 
A

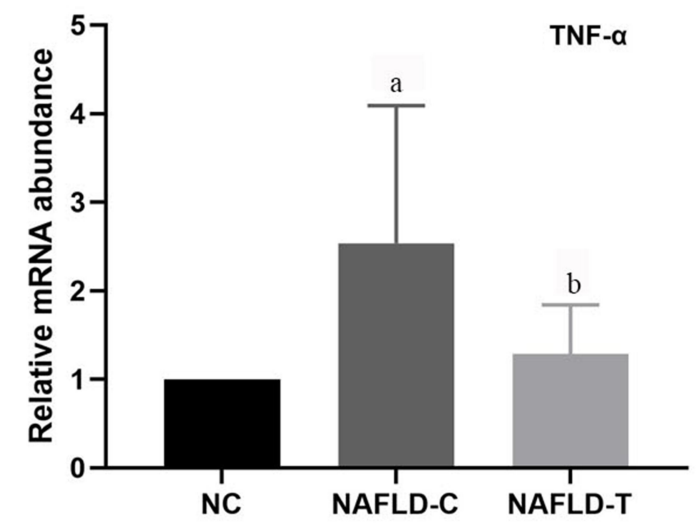

C

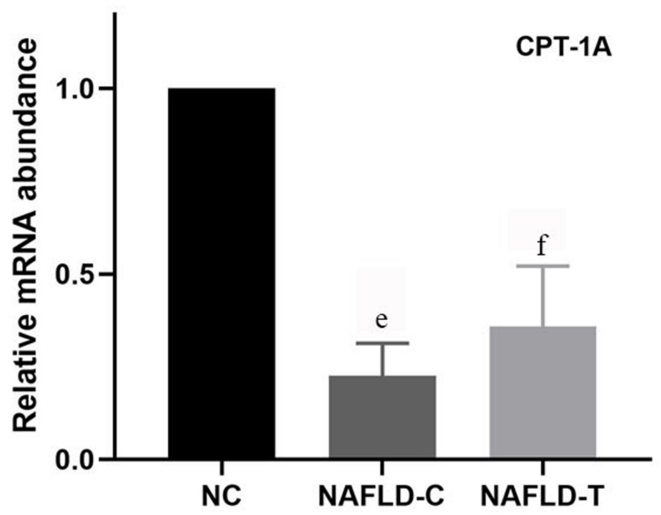

E

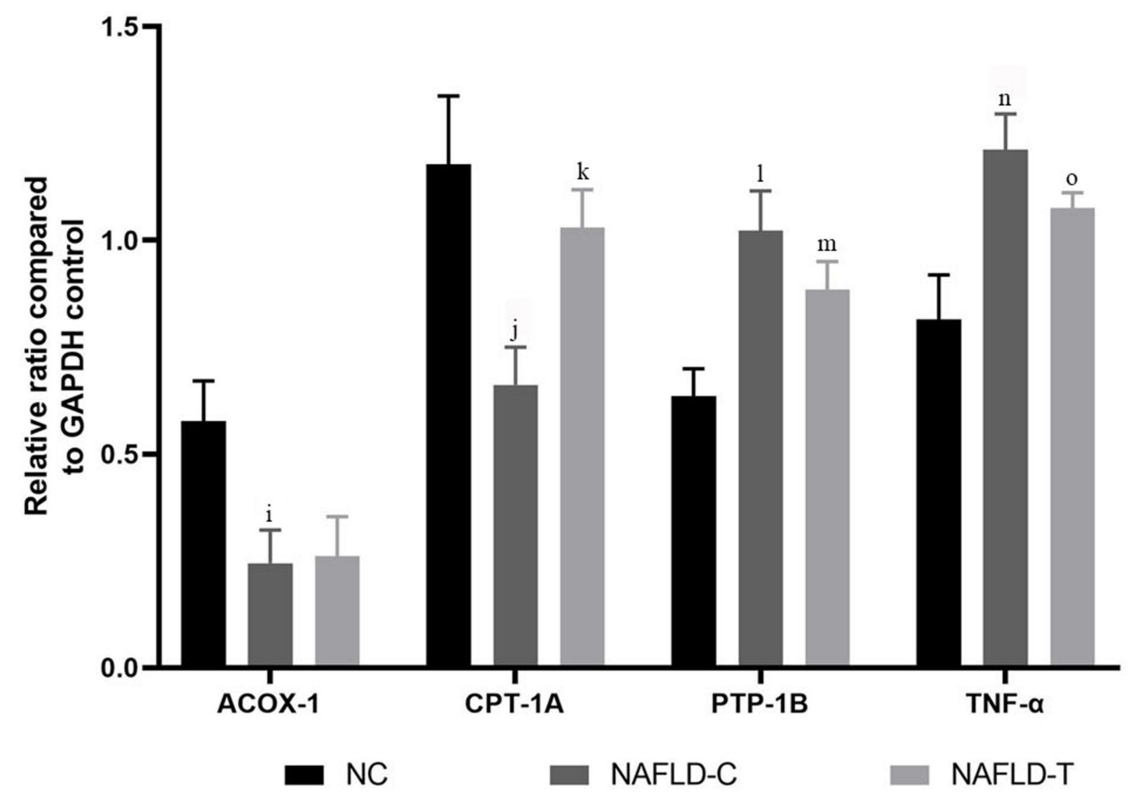

B

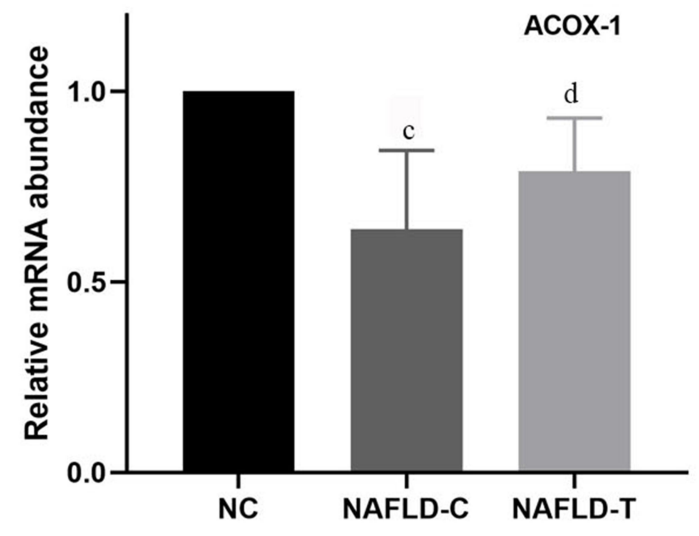

D

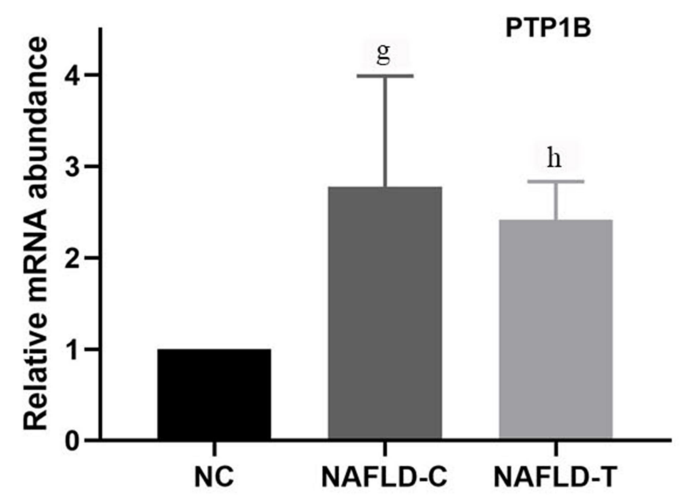

F

Figure 6 Effect of DPP4i treatment on liver (A) TNF- $\alpha$ mRNA expression, (B) ACOX-I mRNA expression, (C) CPT-IA mRNA expression, (D) PTPIB mRNA expression, (E) Effect of DPP4i treatment on liver ACOX-I, CPT-IA, PTP IB, TNF- $\alpha$ protein expression; (F) Western blot analysis of liver ACOX-I, CPT-IA, PTPIB, TNF- $\alpha$ expression. a: NAFLD-C group vs. NC group $P<0.05$; b: NAFLD-C group vs. NAFLD-T group $P<0.05$; c: NAFLD-C group vs. NC group $P<0.05$; d: no significant difference between NAFLD-C and NAFLD-T group; e: NAFLD-C group vs. NC group P< 0.05; f: NAFLD-C group vs. NAFLD-T group $P<0.05$; g: NAFLD-C group vs. NC group $P<0.05$; h: NAFLD-C group vs. NAFLD-T group $P<0.05$; i: NAFLD-C group vs. NC group $P<0.05$; j: NAFLD-C group vs. NC group $P<0.05$; $k$ : NAFLD-C group vs. NAFLD-T group $P<0.05$; I: NAFLD-C group vs. NC group $P<0.05 ;$ m: NAFLD-C group vs. NAFLD-T group $P<0.05$; $n$ : NAFLD-C group vs. NC group $P<0.05$; o: NAFLD-C group vs. NAFLD-T group $P<0.05$. 
infiltrating immune cells and resident liver cells overproduce $T N F-\alpha . T N F-\alpha$ mediated signaling pathway enhancement followed by the activation of kupffer cells may be crucially involved in NASH related liver fibrosis. ${ }^{29}$ Fen et al found that DPP4i linagliptin could lead to macrophage polarization toward an anti-inflammatory phenotype in white adipose tissue and liver, thereby attenuating nonalcoholic steatohepatitis and IR. It further proves the therapeutic effect of DPP4i in NAFLD. ${ }^{30}$

Protein tyrosine phosphatase $1 B(P T P 1 B)$ is deemed as a negative modulator of hepatic insulin signaling pathway, accumulating data indicates that overexpression and hyperactivity of $P T P 1 B$ is related to IR. ${ }^{31,32}$ NAFLD patients normally exhibited elevated $P T P 1 B$ in the liver biopsy. ${ }^{33}$ Current data indicated that HFD could increase $P T P 1 B$ mRNA and protein level, PTP1B reduction was also observed after saxagliptin treatment. It can be concluded that the improvement of DPP4 $i$ is associated with ERS and subsequent apoptosis, mediated by $P T P 1 B$ related pathways, ${ }^{34}$ however, the concrete mechanism needs to be studied further.

DPP4 is a multifunctional serine protease participating in the development of various liver disease including NAFLD. DPP4 exists in two forms: a membrane localised protein and a soluble circulating protein extensively distributing in plasma and body fluids, both of which exert catalytic activity. ${ }^{35}$ DPP4 plays an auxo-action for the occurrence and severity of NAFLD, ${ }^{10}$ moreover, DPP4 activity is proposed as a novel candidate with potential functions in the pathogenesis and histopathologic grade of NASH. ${ }^{10,36}$ NAFLD patients conventionally exert enhanced liver DPP4 expression and activity, ${ }^{37}$ furthermore, high plasma soluble dipeptidyl peptidase 4 (sDPP4) is another typical manifestation among NAFLD patients. ${ }^{10,38,39}$ Our study showed that in addition to serum insulin, both liver DPP4 expression and sDPP4 level are higher in NAFLD mice, which dropped significantly after the administration of saxagliptin. Hepatic lipotoxicity derived from overaccumulation of free fatty acids, hyperinsulinemia, as well as chronic inflammation mediated by $T N F-\alpha$, all of which stimulated hepatocytes to overproduce DPP4 and secrete SDPP4, DPP4 and SDPP4 then in turn deteriorated inflammation and IR. In this respect, DPP4i ameliorates hepatic steatosis by reducing hepatic DPP4 and sDPP4.

Despite the common glucose-lowering effect, different kinds of DPP4i exhibit varying degrees of therapeutic effect of NAFLD. It has been reported that comparing with sitagliptin, linagliptin showed a better capacity of attenuating
NAFLD related inflammation and IR. ${ }^{30}$ There is a possibility that the differences between categories stem from clinical pharmacokinetics and relevant pharmacology. In addition to a much shorter half life and oral bioavailability, saxagliptin is the only substrate for hepatic cytochrome P450 enzymes, in contrast, linagliptin tends to localize in tissues containing high DPP4 levels in rodent models. ${ }^{40}$

In conclusion, saxagliptin improves NAFLD by ameliorating IR, inflammation, downregulation of hepatic DPP4 and sDPP4, as well as subsequent steatosis. The elevation of hepatic DPP4 and sDPP4 and succedent post-treatment decrease suggested that DPP4 may involve in the development of NAFLD. The anti-lipotoxic effect of DPP4i may involve the activation of $C P T 1 A$ and $A C O X 1$ related $\beta$-oxidation signaling pathway, suppression of $T N F-\alpha$ mediated inflammatory and $P T P-1 B$. The results covered in this article showed that saxagliptin affects many aspects of the pathological characteristics of NAFLD, suggesting that DPP4i saxagliptin may offer a novel therapeutic option for NAFLD.

\section{Acknowledgments}

The authors have no ethical conflicts to disclose.

\section{Funding}

This research was funded by grants from the Key Research and Development Plan of Shandong Province (2017G006006 and 2016GSF201016) and National Natural Science Foundation of China (81000325 and 81970700).

\section{Disclosure}

None of the authors have potential conflicts of interest associated with this research.

\section{References}

1. Younossi ZM, Koenig AB, Abdelatif D, et al. Global epidemiology of nonalcoholic fatty liver disease - meta-analytic assessment of prevalence, incidence, and outcomes. Hepatology. 2016;64(1):73-84. doi:10.1002/hep. 28431

2. Ogden CL, Carroll MD, Kit BK, Flegal KM. Prevalence of childhood and adult obesity in the United States, 2011-2012. JAMA. 2014;311 (8):806-814. doi:10.1001/jama.2014.732

3. Feng Z, Jianghua Z, Wang W. Unexpected Rapid Increase in the Burden of NAFLD in China From 2008 to 2018: A Systematic Review and Meta-Analysis.[J]. Hepatology. 2019;70(4):1119-1133. doi:10.1002/hep.30702

4. Estes C, Anstee QM, Arias-Loste MT, et al. Modeling NAFLD disease burden in China, France, Germany, Italy, Japan, Spain, United Kingdom, and United States for the period 2016-2030. J Hepatol. 2018;69(4):896-904. doi:10.1016/j.jhep.2018.05.036 
5. Wenjie D, Ye L, A L, et al. Prevalence of nonalcoholic fatty liver disease in patients with type 2 diabetes mellitus: A meta-analysis.[J] Medicine. 2017;96(39):e8179. doi:10.1097/MD.0000000000008179

6. Brunt Elizabeth M, W-S WV, Nobili V, et al. Nonalcoholic fatty liver disease.[J]. Nat Rev Dis Primers. 2015;1:15080.

7. C R MR, Watt Matthew J. Hepatokines: linking nonalcoholic fatty liver disease and insulin resistance. Nat Rev Endocrinol. 2017;13 (9):509-520. doi:10.1038/nrendo.2017.56

8. Ao N, Jing Y, Wang X, et al. Glucagon-like peptide-1 preserves nonalcoholic fatty liver disease through inhibition of the endoplasmic reticulum stress-associated pathway.[J]. Hepatol Res. 2016;46 (4):343-353. doi:10.1111/hepr.12551

9. Minoru I, Takumi K, et al. Dipeptidyl peptidase-4: a key player in chronic liver disease.[J]. World J Gastroenterol. 2013;19(15):22982306. doi:10.3748/wjg.v19.i15.2298

10. Balaban YH, Korkusuz P, Simsek H, et al. Dipeptidyl peptidase IV (DDP IV) in NASH patients. Ann Hepatol. 2007;6(4):242-250. doi:10.1016/S1665-2681(19)31905-2

11. Xin S, Kong Y, Daoquan P. Fibroblast growth factor 21 in lipid metabolism and non-alcoholic fatty liver disease.[J]. Clin Chim Acta. 2019;498:30-37. doi:10.1016/j.cca.2019.08.005

12. Kovalic Alexander J, Satapathy Sanjaya K, Naga C. Targeting incretin hormones and the ASK-1 pathway as therapeutic options in the treatment of non-alcoholic steatohepatitis.[J]. Hepatol Int. 2018;12 (2):97-106. doi:10.1007/s12072-018-9854-1

13. Carbone Laura J, Angus Peter W, Yeomans Neville D. Incretin-based therapies for the treatment of non-alcoholic fatty liver disease: A systematic review and meta-analysis. $J$ Gastroenterol Hepatol. 2016;31(1):23-31. doi:10.1111/jgh.13026

14. Juan-Juan L, Ping Z. The efficacy of saxagliptin in T2DM patients with non-alcoholic fatty liver disease: preliminary data.[J]. Rev Assoc Med Bras. 1992;2019(65):33-37.

15. Wang Q, Guan XQ. Improvement of induction method of non-alcoholic fatty liver model in rats. Shijie Huaren Xiaohua Zazhi. 2007;15 (11):1219-1224.

16. J K C L, Zhang X, Yu J. Animal models of non-alcoholic fatty liver disease: current perspectives and recent advances[J]. J Pathol. 2017;1:154.

17. Yoshio S, Yuya S, Yoneda M, et al. Novel antidiabetic medications for non-alcoholic fatty liver disease with type 2 diabetes mellitus.[J]. Hepatol Res. 2017;47(4):266-280. doi:10.1111/hepr.12856

18. Browning Jeffrey D, Horton Jay D. Molecular mediators of hepatic steatosis and liver injury. J Clin Invest. 2004;114(2):147-152. doi:10.1172/JCI200422422

19. Christian B, Luisa S, Saussenthaler S, et al. Elevated hepatic DPP4 activity promotes insulin resistance and non-alcoholic fatty liver disease.[J]. Mol Metab. 2017;6(10):1254-1263. doi:10.1016/j.molmet.2017.07.016

20. Ahmad H, Dyah P, Kalista Kemal F, et al. The influence of insulin resistance in the occurence of non-alcoholic fatty liver disease among first degree relatives of type 2 diabetes.[J]. Diabetes Metab Syndr. 2019;13(2):1431-1435. doi:10.1016/j.dsx.2019.01.058

21. Katharina H, Pras-Raves Mia L, Ferdinandusse S, et al. Functional characterisation of peroxisomal $\beta$-oxidation disorders in fibroblasts using lipidomics.[J]. J Inherit Metab Dis. 2018;41(3):479-487. doi:10.1007/s10545-017-0076-9

22. Yu-Pei Z, Yuan-Jun D, et al. Berberine Ameliorates High-Fat DietInduced Non-Alcoholic Fatty Liver Disease in Rats via Activation of SIRT3/AMPK/ACC Pathway.[J]. Curr Med Sci. 2019;39(1):37-43. doi:10.1007/s11596-019-1997-3

23. Sudhir K, Richa R, Karns R, et al. Augmenter of liver regeneration protein deficiency promotes hepatic steatosis by inducing oxidative stress and microRNA-540 expression.[J]. FASEB J. 2019;33 (3):3825-3840. doi:10.1096/fj.201802015R

24. Maja S-R, German P, Mantell Benjamin S, et al. A moderate increase in carnitine palmitoyltransferase $1 \mathrm{a}$ activity is sufficient to substantially reduce hepatic triglyceride levels.[J]. Am J Physiol Endocrinol Metab. 2008;294(5):E969-77. doi:10.1152/ajpendo.00497.2007
25. Cherkaoui-Malki M, Surapureddi S, El Hajj HI, Vamecq J, Andreoletti P. Hepatic steatosis and peroxisomal fatty acid betaoxidation. Curr Drug Metab. 2012;13(10):1412-1421. doi:10.2174/ 138920012803762765

26. Purnomo Hery D. Combination of Aspartate Aminotranferase and Tumor Necrosis Factor- $\alpha$ as Non Invasive Diagnostic Tools for Non Alcoholic Steatohepatitis (NASH).[J]. Acta Med Indones. 2015;47:16-23.

27. Lingyan Y, Zheng C, Lai X, et al. Niacin Ameliorates Hepatic Steatosis by Inhibiting De Novo Lipogenesis Via a GPR109AMediated PKC-ERK1/2-AMPK Signaling Pathway in C57BL/6 Mice Fed a High-Fat Diet.[J]. J Nutr. 2019;1.

28. Frank T, Tom L, Christian T. Inflammatory pathways in liver homeostasis and liver injury. Clin Rev Allergy Immunol. 2009;36:4-12. doi:10.1007/s12016-008-8091-0

29. Annie-Carole T-T, Landes Susan G, Nguyen V, Novobrantseva TI, Hahn YS. Kuppfer cells trigger nonalcoholic steatohepatitis development in diet-induced mouse model through tumor necrosis factor- $\alpha$ production.[J]. J Biol Chem. 2012;287(48):40161-40172. doi:10.10 74/jbc.M112.417014

30. Fen Z, Ni Y, Nagashimada M. DPP-4 Inhibition by Linagliptin Attenuates Obesity-Related Inflammation and Insulin Resistance by Regulating M1/M2 Macrophage Polarization.[J]. Diabetes. 2016;65 (10):2966-2979. doi:10.2337/db16-0317

31. Jesse B, Haj Fawaz G. Protein-tyrosine phosphatase 1B substrates and metabolic regulation.[J]. Semin Cell Dev Biol. 2015;37:58-65. doi:10.1016/j.semcdb.2014.09.020

32. Nicola A, Dambrino Robert J. Role of PTP1B in POMC neurons during chronic high-fat diet: sex differences in regulation of liver lipids and glucose tolerance.[J]. Am J Physiol Regul Integr Comp Physiol. 2018;314(3):R478-R488. doi:10.1152/ajpregu.00287.2017

33. Sanderson Schuyler O, Smyrk Thomas C. The use of protein tyrosine phosphatase $1 \mathrm{~B}$ and insulin receptor immunostains to differentiate nonalcoholic from alcoholic steatohepatitis in liver biopsy specimens. [J]. Am J Clin Pathol. 2005;123(4):503-509. doi:10.1309/1PX2LMP QUH1EE12U

34. Cynthia L, Deborah V, Hazari Y, Hetz C, Chevet E, Bailly-Maitre B. Endoplasmic reticulum stress signalling and the pathogenesis of nonalcoholic fatty liver disease.[J]. J Hepatol. 2018;69(4):927-947. doi:10.1016/j.jhep.2018.06.008

35. Mulvihill Erin E, Drucker Daniel J. Pharmacology, physiology, and mechanisms of action of dipeptidyl peptidase-4 inhibitors.[J]. Endocr Rev. 2014;35:992-1019.

36. Firneisz G, Varga T, Lengyel G, et al. Serum dipeptidyl peptidase-4 activity in insulin resistant patients with non-alcoholic fatty liver disease: a novel liver disease biomarker. PLoS One. 2010;5(8): e12226. doi:10.1371/journal.pone.0012226

37. Masayuki M, Masaki K. Increased hepatic expression of dipeptidyl peptidase-4 in non-alcoholic fatty liver disease and its association with insulin resistance and glucose metabolism.[J]. Mol Med Rep. 2012;5:729-733.

38. Ghorpade Devram S, Lale O, Zheng Z, et al. Hepatocyte-secreted DPP4 in obesity promotes adipose inflammation and insulin resistance.[J]. Nature. 2018;555(7698):673-677. doi:10.1038/nature26138

39. Yoshimasa A, Kanako K, et al. Impact of dapagliflozin, an SGLT2 inhibitor, on serum levels of soluble dipeptidyl peptidase-4 in patients with type 2 diabetes and non-alcoholic fatty liver disease.[J]. Int $J$ Clin Pract. 2019;73(5):e13335. doi:10.1111/ijcp.13335

40. Golightly Larry K, Drayna Caitlin C, McDermott Michael T. Comparative clinical pharmacokinetics of dipeptidyl peptidase-4 inhibitors.[J]. Clin Pharmacokinet. 2012;51(8):501-514. doi:10.10 07/BF03261927 


\section{Publish your work in this journal}

Diabetes, Metabolic Syndrome and Obesity: Targets and Therapy is an international, peer-reviewed open-access journal committed to the rapid publication of the latest laboratory and clinical findings in the fields of diabetes, metabolic syndrome and obesity research. Original research, review, case reports, hypothesis formation, expert opinion and commentaries are all considered for publication. The manuscript management system is completely online and includes a very quick and fair peer-review system, which is all easy to use. Visit http://www.dovepress.com/testimonials.php to read real quotes from published authors.

Submit your manuscript here: https://www.dovepress.com/diabetes-metabolic-syndrome-and-obesity-targets-and-therapy-journal 